\title{
EL ROMANCERO DE GUADALCANAL. UN SIGLO DE TRADICIÓN: DE MICRÓFILO A HOY.
}

\author{
Pedro M. Piñero \\ Virtudes Atero
}

Hace ya algunos años, comenzadas nuestras investigaciones romancísticas por tierras andaluzas, propusimos a Juan Collantes que realizara la encuesta de Guadalcanal y que comparara los textos de la tradición actual con los que en su día había recolectado Micrófilo. Guadalcanal fue reposo poético y refugio familiar para Juan Collantes durante muchos años, y él conocía mejor que nadie sus tradiciones. Su fina sensibilidad poética y sus conocimientos en este campo nos hubieran deparado un estudio, sin duda, inestimable de esta tradición romancística, pero por unas razones u otras no llevó a cabo esta investigación. Nosotros ahora, en su memoria, publicamos estas notas que creemos pueden servir de introducción a un estudio más reposado del romancero de Guadalcanal y de su entorno.

En pleno auge de las investigaciones de los folkloristas de las últimas décadas del siglo pasado, don Juan Antonio Torres Salvador, Micrófilo, constituyó, el 4 de mayo de 1884, la Sociedad El Folk-lore de Guadalcanal. Micrófilo pertenecía al grupo de folkloristas que don Antonio Machado y Álvarez, Demófilo, presidía. Con Guichot y Sierra, Rodríguez Marín, Hernández de Soto y otros colaboraba en las tareas que llevaron a cabo en estas tierras meridionales, muchos de cuyos trabajos fueron apareciendo en El Folk-lore Andaluz, El Folk-lore Frexnense y Bético-Extremeño y en otras revistas y publicaciones que, por aquellos años, fueron los órganos de difusión de estos pioneros del folklore peninsular.

Años después de la aparición de estas revistas, Micrófilo dio a la estampa Un capitulo del Folk-lore Guadalcanalense ${ }^{1}$. En sus páginas daba cuenta de di-

1 Un capitulo del Folk-lore guadalcanalense por Micrófilo, Sevilla, Francisco Leal y Ca, Editores, 1891. XVIII + 151 págs. El libro contiene tres capítulos o partes con sus correspondientes notas: «Rimas infantiles», págs. 19-56, «Botifora», págs. 57-68, y «Romances», págs. 69-151. 
versos aspectos de la cultura popular de este enclave, situado al norte de la provincia de Sevilla, en la raya extremeña. Allí publicó una serie de romances conservados, por aquellas fechas, por los guadalcanalenses. A nuestro modo de ver, y según diversas indicaciones del editor a las que más abajo nos referimos, estos textos son transcripciones fieles de lo que realmente permanecía en la tradición del pueblo.

Eran años, estos de las últimas décadas del Ochocientos, en que empezaba a tomar cuerpo la investigación del romancero de la tradición oral moderna, y a nadie se le oculta la importante labor que en este dominio hicieron aquellos primeros y entusiastas folkloristas, aunque sus métodos de investigación y análisis los consideremos hoy más que superados. De cualquier forma, sus trabajos fueron decisivos en la recuperación de esta tradición poética, de modo especial por estas tierras del Sur.

El corpus de romances de Micrófilo está compuesto por ocho textos, de los cuales cuatro pertenecen al romancero tradicional: Blancaflor y Filomena, Albaniña, Delgadina y Gerineldo más La Condesita; y los otros cuatro, aunque más modernos, también están tradicionalizados: La infanticida, Polonia, El corregidor y la Molinera, y La adúltera del cebollero.

Sin pretender, en modo alguno, ser exhaustivos en el análisis de los textos que nos dejó, sí podemos indicar, en un primer acercamiento, ciertos rasgos que nos den el perfil de este romancero conservado en Guadalcanal en las últimas décadas del siglo pasado.

Hay que destacar, en primer lugar, que en los ocho casos se trata siempre de versiones muy completas, y por lo tanto largas y circunstanciadas en algunas secuencias de la intriga, quizá con la excepción de La adúltera del cebollero, que es una versión que resulta algo comprimida en relación con las conservadas hoy en otros enclaves andaluces. No tenemos datos para pensar que sean versiones facticias compuestas por el mismo Micrófilo; de ser así, desde luego nos parece evidente que esos textos publicados en 1891 serían entonces el resultado de poemas elaborados a partir de fragmentos o retazos tomados realmente de la tradición guadalcanalense. En contra de esta posible suposición, de muy poco fundamento, hay que decir que los folkloristas de esta época publicaban, en la mayoría de los casos, sus textos sin adulterarlos. En muchos lugares expresan su disconformidad con el método de los románticos que, como sabemos, sí modificaban los textos recogidos ${ }^{2}$. Por otro lado, en favor de la fiabilidad de estos romances

2 Cfr. sólo lo que Demófilo opinaba sobre la adulteración o «arreglo» de la literatura oral por parte de los románticos. Escribe a este respecto el prof. López Álvarez: «Sin entrar en esta cuestión, decidido con más o menos convencimiento [...] por las nuevas corrientes científicas, lo vemos incorporar el nuevo método a sus trabajos, aparecidos en La Enciclopedia a partir de 1879, trabajos de los cuales, y según propia confesión: «ya era distinto el concepto que tenía de la literatura del pueblo», pues «no era el valor ideológico desentrañar el sentido oculto de sus producciones, sino únicamente probar la importancia de recogerlas fiel y exactamente para ulteriores fines científicos, lo que me preocupaba» («Cartas inéditas de Demófilo a Joaquín Costa», El Folk-lore andaluz, 2a época, 2, 1988, pág. 40. Y el mismo Micrófilo se expresaba así: «Lo que causa verdadera lástima es ver una producción popular corregida por literatos, gente, por lo común, indocta y cursi en grado superlativo, y que semejan cuando tales empresas acometen, al sastre que pretendiera acomodar a las exigencias de sus 
dados a la luz por Micrófilo parece que habla el hecho de que nuestro folklorista conservara rasgos fonéticos y léxicos peculiares del habla de la zona. Estas dudas o reticencias podían asaltar al estudioso de hoy porque en este repertorio no se encuentra ningún texto fragmentado, lo que parece extraño en cualquier encuesta románcística. Por los datos que tenemos, los folkloristas de esta época sólo publicaban textos completos, y éste debió de ser el caso de Micrófilo. De cualquier forma, no es este el único repertorio que nuestro hombre tenía, porque él mismo expresó que poseía varias versiones de esos temas que «sólo varían en levísimas particularidades ${ }^{3}$. Pero el romancero conocido por Micrófilo no fue, sin duda, mucho más amplio, pues las pocas veces en que se ocupó del género repite estas mismas versiones, y cuando habla de otras de distintos temas, siempre casos muy raros, cita versiones recogidas por otros folkloristas contemporáneos.

Como ocurre siempre en los trabajos dados a conocer por estos estudiosos, no hay referencias concretas a los informantes que les facilitaron los textos.

Todos los romances guadalcanalenses publicados por él son habituales en los repertorios andaluces de hoy, con la excepción quizá de El Corregidor y la Molinera, del que sólo hallamos, por lo general, fragmentos sueltos. También podía destacarse que en algunos casos la actualización de la fábula no se realiza conforme es usual en la tradición andaluza contemporánea. Nos referimos al desenlace de Blancaflor y Filomena, que cuenta con una secuencia extrañamente circunstanciada de la muerte del violador de Filomena, Tarquino en esta versión, que culmina con una sentencia ejemplarizante:
Se levantó Blancaflor,
Como una leona fiera.
Le ha dado de puñaladas,
Le ha sacado la lengua,
La ha puesto por las esquinas
Para que escarmiento sea,
Para que ningún atrevido
Desgonsare á una donseya.

En Delgadina es la madre la que finalmente decide auxiliarla, lo que tampoco es frecuente en la tradición meridional actual del romance, en la que, como se sabe, es el padre el que al final le levanta el castigo, unas veces porque ella accede a sus requerimientos sin fuerzas para continuar en su situación extrema, otras, movido por la piedad. De todas formas, tanto en la versión de Micrófilo de 1891 como en las actuales, el auxilio llega tarde, y Delgadina muere.

Por último, en el caso de Gerineldo, que recogió continuado por La Condesita, según viene siendo normal en Andalucía, lo que ha ocurrido es que el final de Gerineldo, con la referencia al juramento que el paje hace a la Virgen de la

figurines los indóciles miembros de un jayán. Y así les resulta ello. Los salvajes de la Polinesia, vestidos con un sombrero de copa, una faja de general europeo y una estera en los riñones, son Apolos de Belveder al lado de las producciones populares arregladas por literatos». (Un capítulo del Folklore guadalcanalense, ob. cit., pág. 128).

3 Ob. cit., pág. 118. 
Estrella de no casarse con ninguna mujer a la que antes hubiese conocido, se ha desplazado al desenlace de La Condesita, con lo que la historia cambia radicalmente de sentido; mientras que en toda la tradición moderna los amantes terminan unidos, aquí después del reencuentro, Gerineldo —el conde Sol de otras versiones - rechaza a la princesa. Este desplazamiento del motivo provoca un final diferente al del Gerineldo meridional —nos referimos al romance de Gerineldo autónomo, antes de ser continuado por La Condesita-, ya que el texto de Micrófilo termina con una de las fórmulas típicas del noroeste peninsular con la que el paje humildemente reconoce su osadía y pide al rey un castigo: «-Dame la muerte, güen rey/ que bien me la he merecido». Así, mientras que en las versiones norteñas que emplean esta fórmula para el desenlace se sigue la decisión del rey de casar a los amantes, en el texto guadalcanalense el rey vuelve a repetir lo ya dicho en su soliloquio al descubrir a los amantes dormidos: «-No te mato, Gerineldo,/ que te crié desde niño...», ya que no puede darse el matrimonio de la princesa con el paje en este momento, pues entonces la historia no tendría sentido con la continuación que se hace en La Condesita, en donde, como ya hemos dicho, el paje, al final, se niega a casarse con la mujer de la que ya ha gozado.

El repertorio se completa con unas notas del propio Micrófilo muy dentro de la línea menos crítica de los estudiosos del XIX, en un lenguaje marcadamente retórico. Relaciona a veces sus textos con los de Rodríguez Marín; en otros casos, siguiendo a Menéndez Pelayo, señala conocidas fuentes de los temas; o destaca, como otros estudiosos, correspondencias de algunos de los romances con cuentos tradicionales. De todas formas, su crítica es sumamente superficial y en modo alguno original, glosando sin necesidad la fábula de algunos romances o haciendo valoraciones de carácter moral ${ }^{4}$.

Con estos datos previos programamos la encuesta de Guadalcanal, que llevamos a cabo en los días 4 y 5 de marzo de 1988. Nos interesaba no sólo el trabajo de campo como otras tantas veces para continuar completando los enclaves sevillanos, sino también verificar hasta qué punto y de qué manera la tradición actual conserva temas y versiones recogidas cien años antes. Era un buen ejercicio para ver en qué situación se encuentra el romancero por estas zonas. De modo que comenzamos siempre nuestra indagación con los distintos informantes que nos atendieron preguntándoles por los temas que Micrófilo había publicado. No tuvimos mucha suerte al principio. A la crudeza de un tiempo extremadamente frío en la primavera incipiente de la sierra norte, se unía la soledad de las calles de este bello pueblo y la ignorancia alarmante del romancero de las primeras pesonas a las que nos dirigimos. Era como para desistir, pero no lo hicimos. Estas primeras horas de nuestro trabajo presagiaban lo que después sucedió y vamos a exponer aquí: el olvido en que se encuentra en la actualidad el romancero guadalcanalense. Después de mediodía, todo el mundo en el pueblo sabía de nuestra presencia y a lo que íbamos, y así fueron apareciendo las primeras informantes que recordaban algo.

4 Cfr. por ejemplo, Ibídem, págs. 110-111. 
Desde el punto de vista sociológico, conviene señalar que los transmisores de Guadalcanal pueden estar agrupados según dos niveles socioeconómicos y culturales. Un primer grupo está formado por María Romero Bernabé, de 72 años, casada con Rafael Parrón Calderón, que regentan la posada del pueblo, y María Cumbre Carmona, de 70 años, ambas incluidas en un nivel medio-bajo y de una cultura básica que le habían dado unos años de escuela, si bien la mayor parte de la niñez y la juventud la habían pasado en el campo. De ambas conseguimos lo mejor y más rico del romancero de este pueblo, de modo muy especial de $\mathrm{Ma}$ ría Cumbre, cuyo cancionero es mucho más interesante que el de cualquier otra persona de las encuestadas allí, no sólo por sus temas más inusuales en Andalucía, sino también por sus más completas versiones y la variedad de su repertorio.

El segundo grupo de informantes está compuesto por Dolores Calderón, de 59 años, Benigno Riaño, de 70, esposo de la anterior, Josefa Blández, de 68, Encarnación Parrón, de 64, Beatriz Nogales, de 70, Amalia López, de 48, y sus hijas Amalia y Carmen Rivero López, de 22 y 8 años respectivamente. Todos estos forman parte de lo que pudieramos llamar la clase media acomodada y con un nivel cultural aceptable. El romancero que nos cantaron en diferentes sesiones de trabajo puede servir muy bien de exponente de lo que realmente se conserva y, lo que nos parece más importante, del modo cómo ha operado la tradición a lo largo de este siglo en el repertorio romancístico de Guadalcanal. Con el material recogido de ambos grupos creemos que estamos en disposición de ofrecer un primer análisis del romancero guadalcanalense actual.

Hay que resaltar, en primer lugar, que todos cantaban un reducido grupo de romances que se ha conservado en el último estadio de tradicionalización del género, esto es, el romance reducido a canción de corro. Por lo general, estos textos presentan la fábula del modo más esquemático posible y su melodía, casi siempre apoyada con estribillo, es la apropiada para la canción infantil y prevalece sobre la letra. Todas las informantes recuerdan que han utilizado para sus entretenimientos estas cancioncillas, y las niñas nos reafirmaron que todavía hoy las cantan en el corro o en el juego de la comba. No es frecuente, según los datos que poseemos hasta ahora, que no son pocos, que el romance conserve todavía su vitalidad y fluya de modo espontáneo en los juegos infantiles, y esta rareza podemos destacarla como un rasgo peculiar de la tradición romancística de este enclave, aunque en general su romancero resulte hoy tan pobre. Estos son los temas que todavía cantan las niñas: Las señas del esposo, ¿Dónde vas, Alfonso XII?, Don Gato, La viudita del Conde Laurel, Mambrú, Las hijas de Merino, La doncella guerrera (en su versión corta), Los tres alpinos y Las tres cautivas. Como se ve, estos temas son los que comúnmente se encuentran por toda Andaluía, y por toda España, en su modalidad de canción infantil. Pero como queda dicho, la peculiaridad de Guadalcanal es la persistencia de su arraigo a lo largo de distintas generaciones, ya que no sólo los cantan las niñas hoy, sino que las más viejas del lugar los recuerdan en su misma función lúdica. De esta manera, este grupo de romances constituye lo que podemos considerar el núcleo más vivo de la tradición del género mejor asentada en el pueblo. 
En segundo lugar, hay que referirse a la serie de temas comunes en Andalucía, que aquí se hallan en su mayoría muy fragmentados. De este grupo sólo grabamos dos versiones completas y distintas de Don Bueso, una de ellas cantada precisamente por el único informante varón, y Madre, a la puerta hay un niño, recordada por casi todos en su manifestación navideña. Los fragmentos recogidos pertenecen a Santa Elena, Casada de lejas tierras, Tamar (unido a Delgadina), Lux Aeterna, La Virgen y el ciego, San Antonio y los pajaritos, La mala suegra y El curita enfermo. A este grupo de romances fragmentados habría que añadir que muchas de las informantes recordaban haber oído en su juventud otros temas, pero que ahora no podían poner en pie. Por ejemplo: Los primos romeros, Mariana Pineda, Delgadina y La bastarda y el segador. Este conjunto de romances, tan desgastados ya y en franco retroceso, nos habla sin embargo de modo claro de una tradición mucho más floreciente en Guadalcanal, aunque estos temas, frente a los primeros citados - los que se mantienen todavía vivos en la tradición en su forma de canción infantil- debieron de gozar de mucha menor difusión.

Creemos, en tercer lugar, que hay que destacar el repertorio cantado por María Cumbre. Esta informante no sólo recordaba los temas más habituales del pueblo -los pertenecientes a los dos grupos anteriores-, sino que también nos facilitó otros romances que deben citarse por su rareza en el enclave, o por su mejor estado de conservación. Es cierto que una buena parte de este romancero lo había aprendido de su abuela, natural de Zalamea la Serena, por lo que algunas de sus versiones son extremeñas. Esto no debe extrañar ya que Guadalcanal en el pasado dividía su término municipal e incluso su casco urbano entre Sevilla y Badajoz. De lo que nos cantó sobresale, por razones distintas, el Bernal Francés y La loba parda. El primero, por tratarse de una versión más larga y narrativa en comparación con las que de este tema hemos reunido de otros enclaves andaluces, que nunca han sido muchas. La loba es un romance muy extraño en la tradición meridional, y ella misma aclaró que lo había oído recitar a su abuela. Las pocas versiones de este tema encontradas en Andalucía llaman la atención de los estudiosos, pues este es un romance difundido sobre todo en tierras extremeñas. Más habituales en Andalucía son El Quintado, La doncella guerrera en su versión no vulgata, El piojo y la pulga y La dovota de San Antonio, que si los citamos aquí es por su buen estado de conservación y porque sólo ella nos los cantó en el pueblo.

A la vista de todo esto, podemos concluir que nuestra encuesta ha perfilado mejor el panorama romancístico de Guadalcanal, aun teniendo en cuenta el estado lamentable en que se encuentran las más de las versiones grabadas allí, según hemos señalado. Llama la atención el hecho de que ninguno de los temas publicados por Micrófilo, que, como decíamos, eran versiones completas, permanezca hoy en la tradición del pueblo, pues sólo María Cumbre, con una memoria sorprendente para su edad, recordaba haber oído Albaniña, Polonia y Gerineldo. Quiere esto decir, desde nuestro punto de vista, que la tradición ha olvidado, parece que definitivamente, estos temas tan bien conservados hace un siglo. Pero nos queda la duda de si Micrófilo desechó, o no consideró como romances, los que se encuentran todavía con gran vitalidad como canciones de corro. Desde luego 
no incluyó ninguno de ellos entre sus Rimas infantiles, con ser estas abundantes en su publicación. Es de todo punto extraño y singular que justamente los temas más recordados hoy y por lo tanto más enraizados en una tradición mejor asegurada, no existieran a finales del siglo XIX, y más si se tiene en cuenta que estas informantes guadalcanalenses cantaban cuando niñas —esto es: por los años veinte muchas de ellas - este romancero infantil, a poca distancia de la fecha en la que Micrófilo publicó su repertorio.

La investigación completa de la Sierra Norte sevillana está todavía en fase de realización. Cuando se lleve a cabo dispondremos, sin duda, de otros datos complementarios para analizar el romancero de Guadalcanal dentro de su contexto zonal más amplio, y sabremos mejor qué significa en sus rasgos más peculiares, si es que existen, en relación con su región natural.

\section{APÉNDICE}

\section{ROMANCES EN SU ESTADO ACTUAL DE CANCIÓN INFANTIL}

\section{Las señas del esposo}

- Soldadito, soldadito, ¿de dónde ha venido usted?

- De la guerra de Melilla, ¿qué se le ha perdido a usted?

- ¿Ha visto usté a mi marido por la guerra alguna vez?

- No, señora, no lo he visto, ni tampoco sé quién es.

- Mi marido es alto, rubio, alto, rubio, coronel,

y en la cinta del sombrero lleva un pañuelo francés;

se lo bordé cuando chica, cuando chica lo bordé, y otro que le estoy bordando y otro que le bordaré.

- Por las señas que usté ha dado, su marido muerto es, se lo llevan para Francia a casa de un coronel.

- Siete años le he esperado y otros siete esperaré, si a los catorce no viene, de luto me vestiré.

- Calla, calla, Isabelita, calla, calla, Isabel,

yo soy tu querido esposo, tú, mi querida mujer.

(Dolores Calderón, Josefa Blández Romero, Encarnación Parrón Díaz y Beatriz Nogales Yánez).

\section{Don Gato}

Estando un señor don Gato sentadito en su tejado ha recibido una carta que si quiere ser casado con una gatita blanca, sobrina de un gato pardo. 
Por ir a ver a la novia se ha caído del tejado; se ha roto cuatro costillas, el espinazo y el rabo. Ya lo llevan a enterrar por las calles del pescado. $\mathrm{Al}$ olor de las sardinas el gato ha resucitado, por eso dice la gente: «Siete vidas tiene un gato».

(Amalia López Fontán, Amalia Rivero López y Carmen Rivero López).

¿Dónde vas, Alfonso XII?

- ¿Dónde vas, Alfonso XII? ¿dónde vas, triste de ti?

- Voy en busca de Mercedes que ayer tarde no la vi.

- Si Mercedes ya se ha muerto, muerta está que yo la vi;

cuatro duques la llevaban por la calle de Madrid.

Su carita era de seda y sus labios de marfil,

y el velo que la cubría era puro carmesí.

Los zapatos que llevaba eran de puro charol,

regalo de don Alfonso el día que se casó.

Los faroles de palacio ya no quieren alumbrar

porque se ha muerto Mercedes y luto quieren guardar.

(Amalia y Carmen Rivero López).

\section{Las hijas de Merino}

Mi abuela tenía un peral

cargado de peras finas,

en la cogolla más alta

cantaba una golondrina.

Por el pico echaba sangre

y por las alas decía:

- Contigo me he de casar aunque me cueste la vida-

A la hora de cenar se perdió la más pequeña;

su padre la fue a buscar

calle arriba, calle abajo.

¿Dónde la vino a encontrar?

En un portalito oscuro

hablando con su galán.

Y el galán que le decía:

- Contigo me he de casar

aunque me cueste la vida-.

(María Romero Bernabé). 


\section{La doncella guerrera}

Un capitán sevillano siete hijos le dio Dios

y tuvo la mala suerte que ninguno fue varón.

Un día la más pequeña le pilló la salvación:

- Padre, me voy a la guerra vestidita de varón.

- Hija, no vaya(s) a la guerra que te pueden conocer

con ese pelo tan largo y tu cara de mujer-.

Pasaron siete años y nadie la conoció,

sólo el hijo del rey que con ella se casó.

(Lourdes Crespo de la Hera).

\section{La viudita del Conde Laurel}

- Yo soy la viudita del Conde Laurel

que quiero casarme y no encuentro con quién.

- Pues siendo tan bella y no encuentra con quién,

le digo, señora, que aquí tiene usted.

(Dolores Calderón, Josefa Blández Romero,

Encarnación Parrón Díaz y Beatriz Nogales Yánez).

\section{Mambrú}

Mambrú se fue a la guerra,

mire usted, mire usted que pena,

Mambrú se fue a la guerra

no sé cuándo vendrá,

do re mi, do re fa,

no sé cuándo vendrá.

Si vendrá por la Pascua,

mire usted, mire usted qué guasa,

o por la Trinidad,

do re mi, do re fa,

o por la Trinidad.

La Trinidad se pasa,

mire usted, mire usted que guasa,

la Trinidad se pasa,

Mambrú no viene ya,

do re mi, do re fa,

Mambrú no viene ya.

Por allí viene un paje,

mire usted, mire usted que traje,

por allí viene un paje, 
¿qué noticias traerá?

do re mi, do re fa,

¿qué noticias traerá?

Las noticias que traigo,

mire usted, mire usted qué traigo,

las noticias que traigo

dan ganas de llorar,

do re mi, do re fa,

dan ganas de llorar.

Que Mambrú ya se ha muerto,

mire usted, mire usted qué tuerto,

que Mambrú ya se ha muerto,

lo llevan a enterrar,

do re mi, do re fa,

lo llevan a enterrar.

Con caja de terciopelo,

mire usted, mire usted qué duelo,

con caja de terciopelo,

con tapa de cristal,

do re mi, do re fa,

con tapa de cristal.

Y encima de la tapa,

mire usted, mire usted qué tapa,

tres pajaritos van,

do re mi, do re fa,

tres pajaritos van.

Cantando el pío-pío,

mire usted, mire usted qué tío,

cantando el pío-pío,

cantando el pío-pa,

do re mi, do re fa,

cantando el pío-pa.

(Amalia Rivero López)

\section{ROMANCERO NO INFANTIL}

a) Versiones completas.

\section{Don Bueso}

- Apártate, mora bella, apártate, mora linda, deja beber mi caballo agua fresca y cristalina.

- No me diga usté a mí mora que soy de España nacida; 
me cautivaron los moros noche de Pascua florida.

- ¿Si quieres volver a España en mi caballo subida?

- Y la ropa que yo lavo ¿dónde la dejo metida?

- La de hilo y la de holanda en mi maleta metida, y la que no valga nada el río abajo se tira.

- Y mi honra, caballero, ¿cuándo la recobraría?

- Yo te juro por mi espada: por mi pecho pasaría-.

Al pasar por unos montes la mora se echó a llorar:

- ¿Por qué lloras, mora bella? ¿por qué lloras, mora linda?

- Porque aquí mis padres a cazar siempre venían,

y mi hermano Moralejo a cazar siempre venía.

- Permita Dios del cielo, Virgen sagrada María, que por traerme una mora, me traje una hermana mía.

(María Romero Bernabé).

b) Versiones fragmentadas.

\section{Santa Elena}

Estando tres niñas bordando corbatas

pasó un caballero pidiendo posada.

- Si mi madre quiere, de muy buena gana-.

Le puso la mesa en medio la sala:

cuchillos de oro, manteles de plata.

Le puso la cama en medio la sala.

//.........................................//

(Benigno Riaño Rivero)

Casada de lejas tierras

- Maridito mío, si tú me quisieras,

a la tuya madre a llamarla fueras-.

- Levántate, madre, del dulce dormir

que la bella Aurora ya quiere parir.

- Si pare, que para, que para un león,

que se le vuelva sangre hasta el corazón.

- Mujercita mía,

(mi madre no puede venir porque está malita.

Coge la jaca y ve a llamar a mi madre que te

estará esperando. La madre vivía en otro pueblo).

- Levántate, suegra, del dulce dormir

que la bella Aurora ya quiere parir. 
- Espérate, yerno, espera en la puerta, espera que coja la ropa y la cesta-.

//..........................................//

(María Romero Bernabé)

\section{La mala suegra}

Carmela se paseaba por una salita alante

con un dolor de cabeza que el corazón se le parte.

/................................................../

- Si a la noche viene Pedro yo le pondré de cenar, y si quiere ropa limpia yo también se la sé dar-.

Por la noche vino Pedro: - Mi Carmela, ¿dónde está?

- Tu Carmela es una tuna, no tiene perdón de Dios

//..............................................//

(Encarnación Parrón Díaz)

\section{ROMANCERO DE MARÍA CUMBRE CARMONA}

\section{La loba parda}

Estando en la mía choza pintando mis alpargatas vi venir siete lobos por lo alto Sierra Nevada;

venían echando suertes a ve(r) a quién le tocaba;

le tocó a una loba chica, patituerta y jorobada.

Le dio una vuelta a la red y no pudo sacar nada,

le dio otra media vuelta, sacó una ovejita blanca, sobrinita de la negra.

Traía siete cachorros y una perra trujillana.

- Andar, perrillos, ahí, andar, perrillos, de fama, si me traéis la borrega la cena tenéis ganada:

un caldero de calostros, otro de leche migada.

- No le temo a tus cachorros, ni a la perra trujillana, que tengo yo mis dientes como puntas de navaja-. Anduvieron siete leguas, todas ellas barbechadas, al pasar el arroyuelo la loba se vio cansada.

- Tome usted su borrega, viva y sana como estaba.

-Yo no quiero mi borrega que la traes maltratada.

Lo que quiero son las orejas pa un abanico que se abanique el ama; lo que quiero son las patas pa un banco pa hacer la matanza;

y la piel pa el pastor una zamarra. 


\section{Bernal Francés}

- Tras, tras, que a la puerta llaman

- Abrir que soy don Francisco a quien tú sueles abrir-.

Bajando por la escalera se le apagó el candil, la ha cogido de la mano y se la llevó al jardín.

Le lavó sus blancas piernas con agua de toronjil, le secó sus blancas piernas con toallas de marfil. Lo ha cogido de la mano y se lo llevó a dormir. A esto de la media noche se le ha ocurrido decir: - ¿Qué tiene usted, don Francisco, que no se vuelve hacia mí? ¿Le teme usted a la justicia o le teme al aguacil? No le tema a la justicia, ni le tema al aguacil, ni le tema mi marido que se fue a tierra de Madrid.

\section{El Quintado}

- El día en que me casé me llevaron a la guerra, y he dejado a mi mujer ni casada ni soltera.

- ¿Tan guapa es tu mujer que tanto te acuerdas de ella?Del bolsillo que llevaba sacó una foto de ella. ¡Mira si sería guapa, mira si sería bella que hasta el mismo capitán se ha enamorado de ella! - Toma la licencia y vete y cuida de esa doncella, que por un soldado menos no se va a perder la guerra-. - Ábreme la puerta, sol, ábreme la puerta, estrella.

- La puerta yo no la abro, mi marido está en la guerra.

- Abreme la puerta, sol, ábreme la puerta, estrella, que por tu cara bonita me he librado de la guerra-. Allí fueron los abrazos y allí fueron los delirios, y allí fueron encontrados los dos esposos perdidos.

\section{La doncella guerrera}

- Malhaya la suerte mía de las siete hijas que tengo y ningún hijo varón-. Y le dice la pequeña: - No eche usted esa maldición; deme usted espada y caballo y a la guerra me voy yo. - Tienes la cara de rosa para ser hombre varón.

- Eso no importa, padre, y a la guerra me voy yo.

- Tienes el cabello largo para ser hombre varón.

- Eso no importa, padre, eso me lo corto yo.

- Tiene(s) el pecho mu abultado para ser hombre varón.

- Eso no importa, padre, eso me lo oculto yo-.

Siete año(s) estuvo sirviendo y nadie se lo notó, 
y una tarde paseando con los reyes de Borbón se le ha caído la espada, por decir: - Dios que pequé; por decir: - Dios que pequé, dijo: - Pecadora yo- Madrecita, la mía reina, que yo me muero de amor que el caballero don Marcos es hembra que no es varón. - Convídalo tú, hijo mío, a correr contigo un día que si ella fuera mujer nunca se cansaría.

- Los tres caballeros, madre, enseguida se cansaron y el caballero don Marcos ha corrido más que un galgo.

- Madrecita, la mía reina, que yo me muero de amor que el caballero don Marcos es hembra que no es varón. - Convídalo tú, hijo mío, a coger peras un día que si ella fuera mujer en la falda se la(s) echaría. - Los tres caballeros, madre, las mangas se las llenaron y el caballero don Marcos

- Convídalo tu, hijo mío, a nada(r) contigo un día que si ella fuera mujer nunca se desnudaría.

- Los tres caballeros, madre, se echaron a desnudar y el caballero don Marcos se ha sentado a llorar.

- ¿Por qué llora usted, don Marcos? - Porque tengo que llorar, porque mi pena es muy grande y no la puedo ocultar-.

La ha cogido de la mano con contento y alegría, le dijo a su madre reina: — Ya logré lo que quería-. 